\title{
Effect of Debris Distribution on Wall Concavity in Deep-Hole EDM*
}

\author{
Serkan CETIN***, Akira OKADA*** and Yoshiyuki UNO***
}

\begin{abstract}
In deep-hole electrical discharge machining, the electrode jump height and hence the movement of the machining debris inside the side gap affect the wall concavity of the machined holes. In this study, the dielectric fluid flow and the debris distribution in the machining gap caused by electrode jump is analyzed to understand the wall concavity phenomenon of the holes using a computational fluid dynamics (CFD) simulation program. Fluid flow and debris-fluid interaction under low and high electrode jumps are examined and compared. The accuracy of the predictions obtained by the numerical calculation is assessed through comparisons with experimental data.
\end{abstract}

Key Words: EDM, Electrode Jump, Dielectric Fluid Flow, Debris Distribution, CFD Simulation

\section{Introduction}

Ejection of debris from the machining gap in electrical discharge machining (EDM) is an important phenomenon in stable and precise machining ${ }^{(1)-(4)}$. It is also well known that various types of flushing methods have been studied, proposed and used to improve the ejection of debris from the gap ${ }^{(5),(6)}$. Fortunately, recent linearmotor-equipped ED machines have shown advances in noflush machining because of the application of their highspeed jump function ${ }^{(7),(8)}$. The high-speed jump function enables a larger amount of dielectric fluid to be pumped in and out of the machining gap, hence causing a decrease of the debris concentration in the machining gap ${ }^{(9)}$. For this reason, the relationship between the flush action of the electrode and the behavior of debris in the gap is of technical interest in linear-motor-equipped EDM.

Koenig et al. ${ }^{(10)}$ described a mathematical model for the flow field at the frontal gap, where the pressure and velocity fields were determined using mathematical functions. Masuzawa et al. ${ }^{(5)}$ simulated the flow field of jet

* Received 23rd October, 2003 (No. 03-4141)

** Graduate School of Natural Science and Technology, Okayama University, 3-1-1 Tsushima-naka, Okayama 700-8530, Japan.

E-mail: cetin@ntmlab.mech.okayama-u.ac.jp

*** Department of Mechanical Engineering, Okayama University, Okayama 700-8530, Japan.

E-mail: okada@mech.okayama-u.ac.jp,

uno@mech.okayama-u.ac.jp flushing and numerically calculated the debris distribution in the gap. Kunieda and Mori ${ }^{(11)}$ calculated the velocity field of debris transport by superposing the radial drift velocity caused by dielectric flushing and the dispersion velocity from the succession of bubble expansion around each discharge. However, all the studies mentioned above involved the examination of the distribution of debris in the bottom gap, along the facing surfaces of the electrode and the workpiece. The relationship between the electrode jump action and debris movement in both bottom and side gaps has not been reported to date. Hence, we investigate the motion and exclusion of debris from the bottom and side gaps during electrode jump, when there is no electrical discharge.

In the first part of this study, the effect of electrode jump height on the wall concavity of the machined holes is discussed. Next, the dielectric fluid flow and the debris distribution in the machining gap resulting from low and high electrode jumps are simulated using a computational fluid dynamics (CFD) simulation program. The wall concavity phenomenon of the holes is explained using the debris distribution schemes obtained from the simulation. Lastly, the accuracy of the predictions of the CFD calculations is assessed through comparisons with experimental data.

\section{Wall Concavity Concept of Deep Holes}

Experiments were conducted with linear-motorequipped ED machines to investigate the effect of electrode jump height on the machined wall concavity. Fig- 


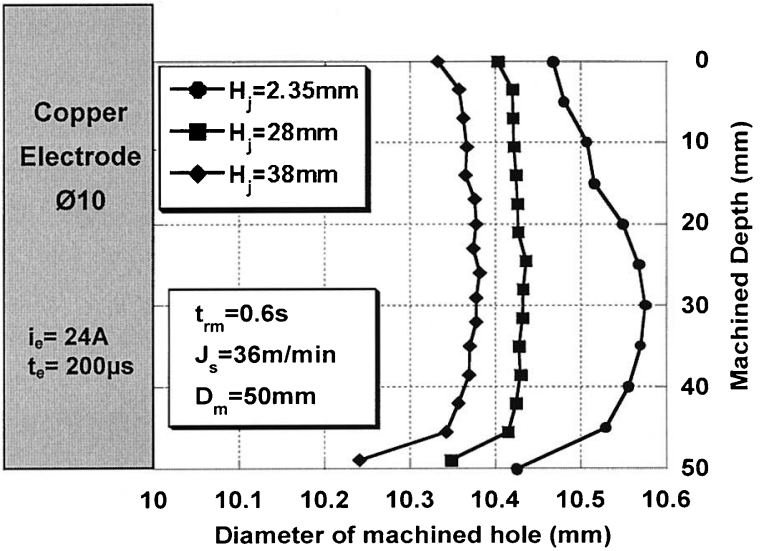

Fig. 1 Jump height vs wall profile of machined hole

ure 1 shows the wall profiles of the machined holes with respect to three different electrode jump heights for a total machining depth of $50 \mathrm{~mm}$. The wall concavity is defined as the difference between the maximum and the average minimum diameter of the wall. In this graph, $H_{j}$ represents the maximum jump height of the electrode; $t_{r m}$ refers to the duration between two successive electrode jumps; $J_{s}$ indicates the maximum speed of the electrode during the jump; and $D_{m}$ represents the machining depth. The graph shows that the electrode jump height affects both the concavity of the side walls and the average machining side gap. The concavity and the average machining side gap become smaller with increasing jump height.

The reason why the average side gap becomes smaller with increasing jump height can be explained in terms of the drop in debris concentration, as mentioned in our past work ${ }^{(9)}$. However, in this paper, the concavity concept of the holes machined with different electrode jump heights will be focused on and will be based on the assumption that some secondary discharges are localized in the side gap. Since the localized secondary discharges are produced by high-debris-concentration regions, the debris movement and the distribution of debris particles in the side gap will be explained with the dielectric fluid flow behavior given in the following sections.

\section{Computational Fluid Flow and Debris Motion}

In this part of the study, the problem is approached using CFD calculations where the electrode jump motion is modeled and then the flow field and debris movement are computationally simulated. The computations presented in this study have been performed using the general-purpose commercial Star-CD code.

\subsection{Electrode jump model}

Figure 2 shows the simplified CFD model, where only one-half of the electrode workpiece is simulated since the other half is symmetrically the same. The electrode in the model is $10 \mathrm{~mm}$ in diameter. It jumps for two different jump heights $\left(H_{j}\right)$ of 2.4 and $38.0 \mathrm{~mm}$ with jump times

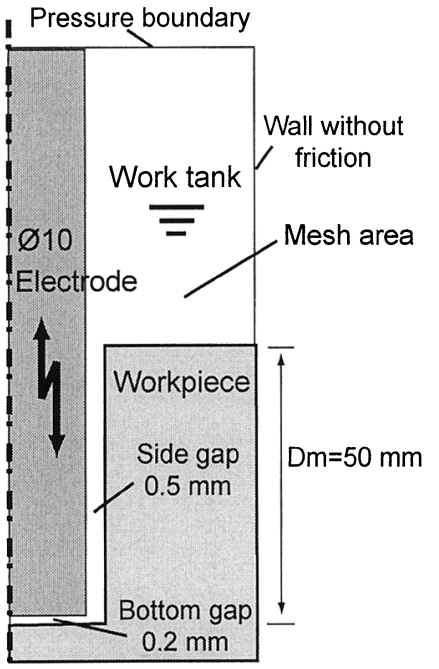

Fig. 2 CFD model for electrode jump

$\left(t_{j}\right)$ of 0.16 and $0.50 \mathrm{~s}$, respectively ( $t_{j}$ indicates the duration required for the electrode to complete one full jump). The jump motion of the electrode is modeled as a sine ${ }^{3}$ wave function representing the real jump movement. The machining depth $\left(D_{m}\right)$ is $50 \mathrm{~mm}$ for both cases. Side and bottom gaps are 0.2 and $0.5 \mathrm{~mm}$, respectively.

In this model, because of the moving boundary of the jumping electrode, the control over mesh resolution and distortion was simulated by "cell-layer removal/addition" and "mesh motion" methods at the bottom and side gaps $^{(12)}$. In the first method, bottom cell layers were enabled to be removed or added during the transient calculation so that the average cell size would remain roughly constant. The cell number in the bottom gap was increased from $110 \times 4$ to $110 \times 28$ cells during the ascending period of the electrode for the jump height of $2.4 \mathrm{~mm}$; whereas the cell number was increased from $110 \times 4$ to $110 \times 384$ cells for jump height of $38.0 \mathrm{~mm}$. In the second method, the side gap cells were programmed so that they could be progressively compressed and expanded during the electrode jump. The side gap consists of $10 \times 1000$ cells in both cases.

The numerical analysis was performed under the assumption that the flow is two-dimensional and turbulent for kerosene fluid with a density of $760 \mathrm{~kg} / \mathrm{m}^{3}$ and kinematic viscosity of $1.881 \times 10^{-6} \mathrm{~m}^{2} / \mathrm{s}$ where the effect of gravity on the flow is neglected. The $K-E$ /high Reynolds number model was chosen for the turbulence model. The turbulence intensity and mixing length values were set to 0.03 and 0.0035 at the pressure boundary (Fig. 2). The main algorithm used was the PISO (pressure implicit with splitting of operators) method with a calculation time step of $0.1 \mathrm{~ms}$.

\subsection{Simulation of fluid flow}

The fluid flow in bottom and side gaps are simulated during the electrode jump since fluid flow is one 


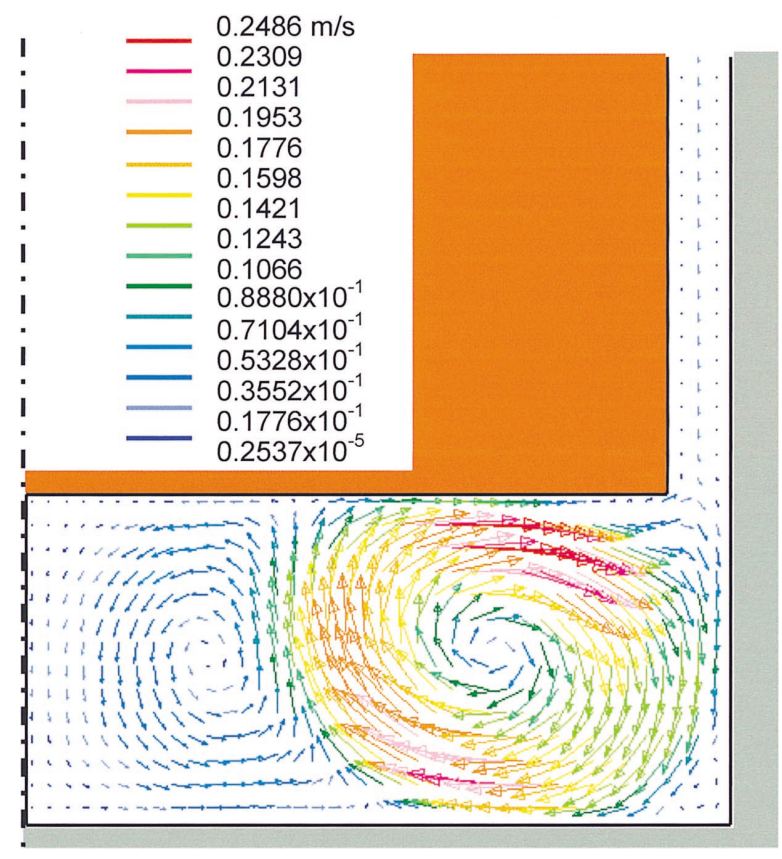

Fig. 3 Velocity field pattern for $H_{j}=2.4 \mathrm{~mm}$ and $t=0.08 \mathrm{~s}$

of the main driving forces of debris distribution. Figure 3 presents the calculated velocity field pattern when the electrode reaches its maximum jump height of $2.4 \mathrm{~mm}$. The figure shows that there are two vortexes generated during the ascending period of a low-jump electrode. The maximum flow speed, at the periphery of the right vortex, is $0.249 \mathrm{~m} / \mathrm{s}$ at $t=0.08 \mathrm{~s}$. Figure 4 presents the calculated velocity field pattern when the electrode jumps up to $38.0 \mathrm{~mm}$. Although there are again two vortexes generated for high electrode jumps, the vortexes are now aligned vertically instead of horizontally, where the upper vortex is much bigger and dominant compared to the lower vortex. The maximum flow speed for the particular electrode position is about $0.269 \mathrm{~m} / \mathrm{s}$. As shown, there is not much difference between the two maximum flow speeds for low and high electrode jumps. This could be explained by the maximum jump speed of the electrode being about $0.6 \mathrm{~m} / \mathrm{s}$ in both cases.

\subsection{Simulation of debris motion}

In the previous section, we showed that the low and high electrode jumps lead to different fluid flow schemes in the jump gap. The debris generated during the discharging process are relocated and/or flushed out by the fluid flow of the jump action. Hence, the interaction between the dielectric fluid and machining debris is of interest.

A Lagrangian/Eulerian kind of framework is used to solve the dispersed two-phase flow during the electrode jump. Figure 5 shows the initial location of the debris particles to be tracked when $t=0 \mathrm{~s}$. The debris particles are numbered, colored and resized for better visualization. The inner-bottom gap, outer-bottom gap, side gap and outside gap (inside work tank) particles are represented by

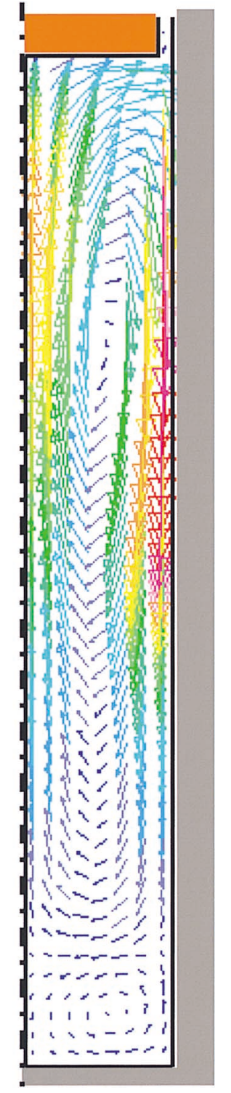

Fig. 4 Velocity field pattern for $H_{j}=38.0 \mathrm{~mm}$ and $t=0.25 \mathrm{~s}$

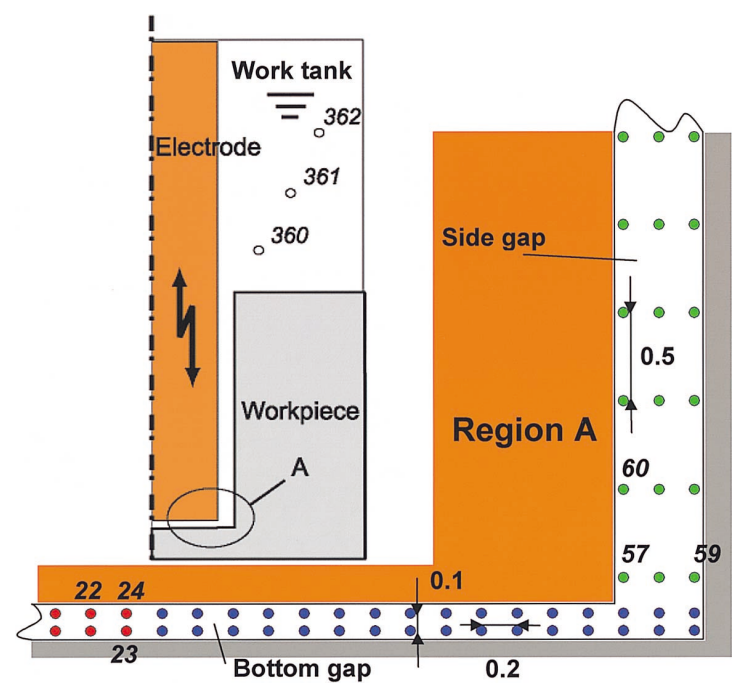

Fig. 5 Initial location of debris particles in bottom gap, side gap, and inside work tank at $t=0 \mathrm{~s}$

red, blue, green and white colors, respectively. Because of the difficulties in identifying the actual debris positions, the debris particles are evenly distributed in the present model. Although in actual machining it is known that the debris particle number and size vary greatly according to the machining conditions, 362 round debris particles were distributed uniformly in the bottom and side gaps with a constant particle diameter and density of $10 \mu \mathrm{m}$ and 
$7.8 \mathrm{~g} / \mathrm{cm}^{3}$.

In this dispersed two-phase flow model, the influence of gravity is again neglected. The momentum transfer process between the two phases is activated so that the motion of the debris will be influenced by the fluid flow and vice versa. The model also accounts for wall bouncing and inter-debris particle collision ${ }^{(12)}$.

\subsubsection{Simulation results of debris motion Fig-} ure 6 shows the debris particle motion in the jump volume (the volume created when the electrode is retracted) for electrode jump conditions of $H_{j}=2.4 \mathrm{~mm}$ and $t_{j}=0.16 \mathrm{~s}$. Figure 6 (a) presents the debris distribution at $t=0.08 \mathrm{~s}$, where the electrode reaches its maximum jump height. Almost all debris particles initially located in the bottom gap (colored in red and blue), are collected near the top center of the electrode and bottom center of the workpiece. On the other hand, some of the debris particles located initially in the side gap (colored in green), have entered the jump volume and interacted with the vortex. Figure 6 (b) shows the debris distribution for $t=0.16 \mathrm{~s}$, at the moment where the electrode completes its full jump. The new debris distribution in the bottom gap shows that most of the red particles remain at the bottom gap, whereas most of the blue particles are ejected from the bottom gap into the side gap. It is also clear that some of the green particles have entered the bottom gap.

The debris distribution in the side gap between depths 0 and $50 \mathrm{~mm}$ at $t=0.16 \mathrm{~s}$ is shown in Fig. 7. The red and blue particles ejected from the bottom gap, and some of the redistributed green particles cause an uneven debris distribution leading to debris-rich regions in the side gap. The debris-rich regions are shown in circles in the figure. It should be noted that the debris-rich regions are mostly between the center and lower part of the side gap, i.e., between 20 and $50 \mathrm{~mm}$.

Figure 8 is the enlarged view of the lower side gap. The figure shows that a vortex is generated when the electrode completes its full jump. This generated vortex is considered to be another reason for the increase of the debris concentration at that specific region because it captures some of the debris particles. In the last section of the paper, the characteristics of the calculated vortex will

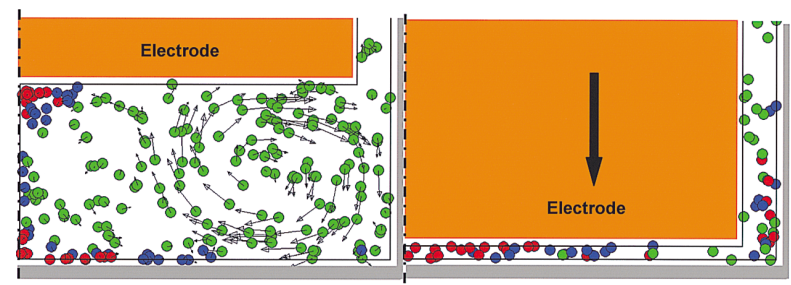

(a) $t=0.08 \mathrm{~s}$

(b) $t=0.16 \mathrm{~s}$

Fig. 6 Debris particles motion during electrode jump for $H_{j}=2.4 \mathrm{~mm}$ and $t_{j}=0.16 \mathrm{~s}$ be investigated experimentally and the correspondence between the flow simulation and experiment results will be confirmed by means of vortex size, center position and start time.

Figure 9 illustrates the debris particle motion for high electrode jump conditions of $H_{j}=38.0 \mathrm{~mm}$ and $t_{j}=0.50 \mathrm{~s}$. Figure 9 (a) presents the debris distribution at $t=0.25 \mathrm{~s}$. Almost all the debris particles presented in the model are carried into the jump volume and mixed by the strong fluid flow of the jump action. Figure 9 (b) shows the debris exclusion from the jump gap at $t=0.35 \mathrm{~s}$. As the electrode descends, the debris particles near the side gap entrance are disposed first; red, blue and green particles are disposed all together. The final debris distribution at $t=0.50 \mathrm{~s}$ is illustrated in Fig. 9 (c). As shown, almost no debris particle is left in the bottom gap (only particle number 11), whereas a few particles remain in the side gap (particle

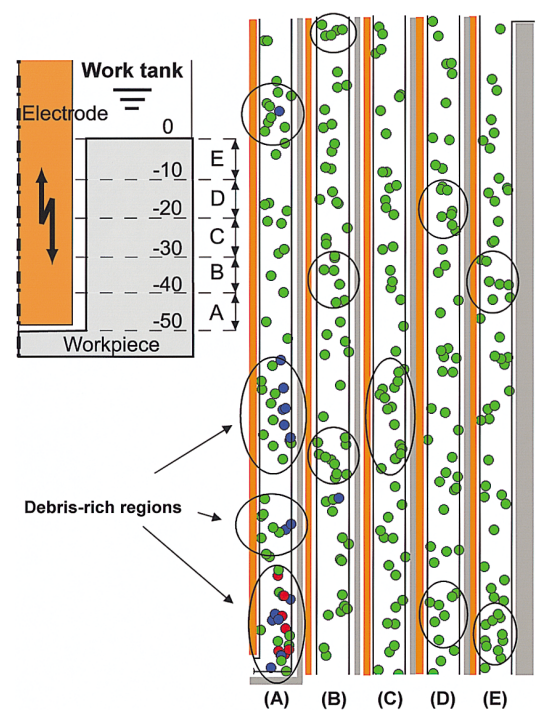

Fig. 7 Debris distribution in side gap from depth 0 to $50 \mathrm{~mm}$ at $t=0.16 \mathrm{~s}$

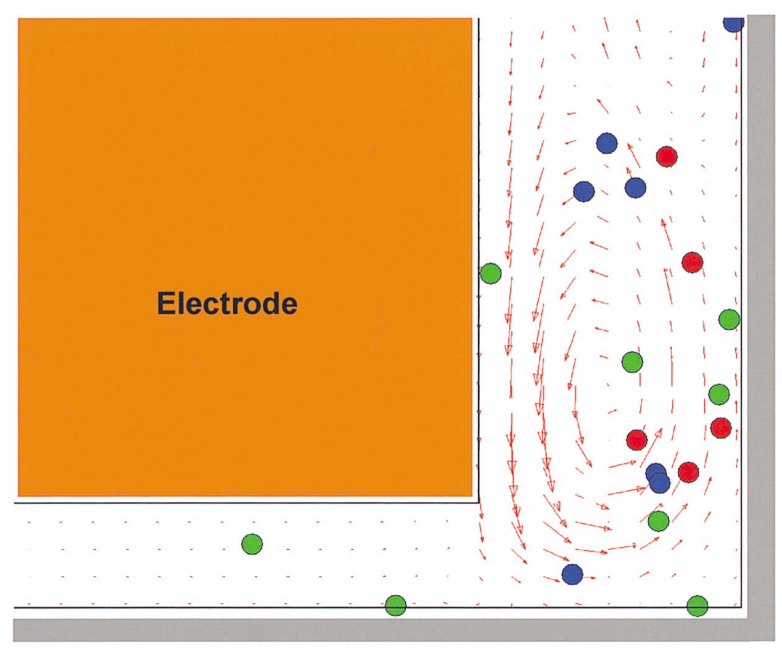

Fig. 8 Vortex generated at $t=0.16 \mathrm{~s}$ captures some of debris particles in that specific region 


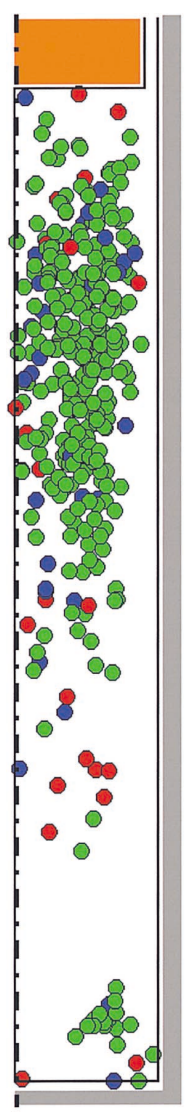

(a) $t=0.25 \mathrm{~s}$

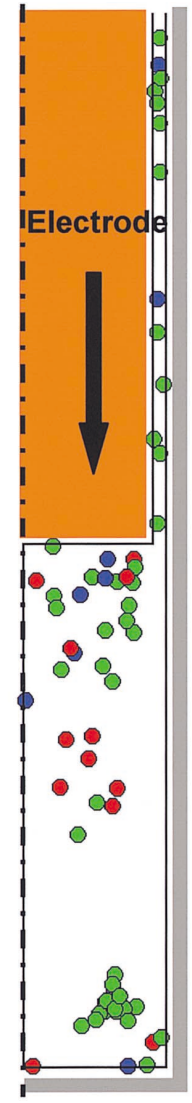

(b) $t=0.35 \mathrm{~s}$



(c) $t=0.50 \mathrm{~s}$
Fig. 9 Debris particle motion during electrode jump for $H_{j}=38.0 \mathrm{~mm}$ and $t_{j}=0.50 \mathrm{~s}$

numbers $7,17,31,67,71,74,77,80,86,91,92,95,155$, $185,248)$. Most of the particles are ejected to the work tank by the high jump flow action.

\section{Experimental Flow Analysis}

\section{1 Experimental setup}

The visual observation of the dielectric fluid in a real machining gap is difficult because of the highly bright electrical discharges and rising bubbles caused by the evaporating dielectric fluid. For this reason, a dummy workpiece-electrode arrangement is separately fabricated to simulate the actual dielectric flow in the machining gap, where the dummy electrode moves with the same real jump parameters in a separate tank (Fig. 10). The dummy electrode is made of square aluminum with a width of $10 \mathrm{~mm}$ which represents the diameter of the real cylindrical copper electrode. The dummy workpiece is produced from acrylic material so that light could pass through the workpiece walls to enable a better visual view of the working gap. Figure 11 presents a schematic illustration of the observed area in the dummy workpiece-electrode arrangement during the electrode jump. The bottom and side gaps are set to 0.2 and $0.5 \mathrm{~mm}$, respectively. The observation area is set to $1 \times 2.2 \mathrm{~mm}$ at the lower side gap. Glass pow-

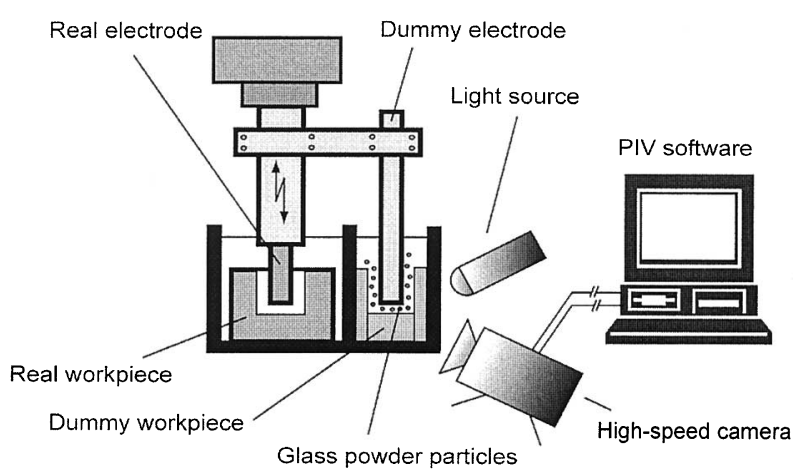

Fig. 10 Experimental setup

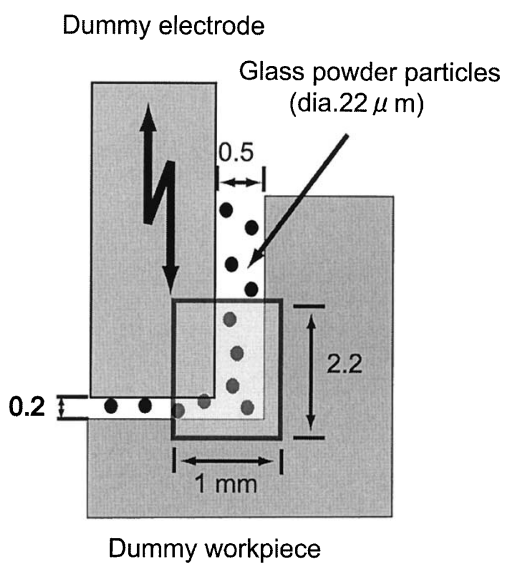

Fig. 11 Region of observation

der particles of about $22 \mu \mathrm{m}$ in diameter were added to obtain a better view of the flow.

In this experimental study, the observation and flow visualization in the model gap were carried out by the PIV (particle image velocimetry) technique. In this technique, the flow images were recorded with a high-speed camera (recording speed of $500 \mathrm{fps}$ ) and then integrated with a PIV software package in order to understand the flow characteristics of the dielectric fluid.

\subsection{Flow field visualization results}

Figure 12 (a) shows the recorded picture of dielectric fluid flow when the electrode just completes its full jump, i.e., $t=0.5 \mathrm{~s}$. As seen from the image, a vortex is generated at the lower side gap for the jump conditions $H_{j}=38 \mathrm{~mm}, J_{s}=36 \mathrm{~m} / \mathrm{min}$, and $t_{j}=0.5 \mathrm{~s}$. Figure $12(\mathrm{~b})$ shows the velocity field pattern of the flow obtained using the PIV software for the same instant and under the same jump conditions.

\section{Comparison of Experimental and Simulated Re- sults}

Table 1 gives the CFD and experimental results, based on some quantitative vortex information for $H_{j}=38 \mathrm{~mm}$ and $t_{j}=0.5 \mathrm{~s}$. The vortex center position gives the approximate $x$ and $y$ coordinates of the vortex center when the electrode completes a full jump. The $x$ and $y$ values 


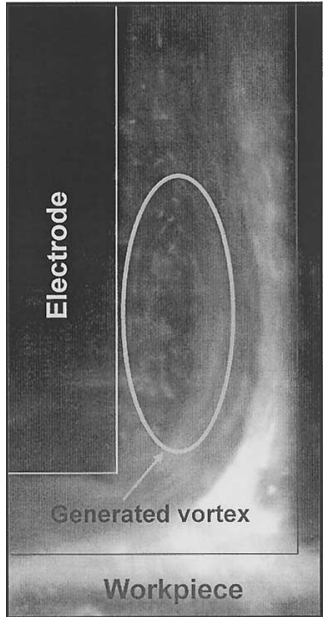

(a) Observation of vortex at $t=0.5 \mathrm{~s}$ for $H_{j}=$ $38 \mathrm{~mm}$

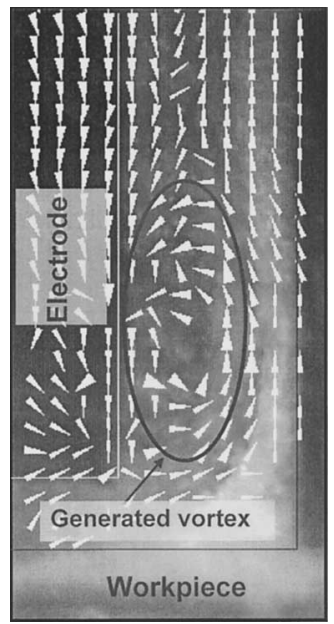

(b) Flow field pattern at $t=0.5 \mathrm{~s}$ for $H_{j}=$ $38 \mathrm{~mm}$
Fig. 12 Observation of vortex and flow field pattern

Table 1 Simulated and experimental results

\begin{tabular}{|l|c|c|}
\hline & $\begin{array}{c}\text { CFD } \\
\text { results }\end{array}$ & $\begin{array}{c}\text { Experimental } \\
\text { results }\end{array}$ \\
\hline Vortex center position $(\mathrm{mm})$ & $\begin{array}{c}\mathrm{x}=5.225 \\
\mathrm{y}=0.450\end{array}$ & $\begin{array}{c}\mathrm{x}=5.225 \\
\mathrm{y}=0.625\end{array}$ \\
\hline Vortex size $(\mathrm{mm})$ & $\begin{array}{c}\mathrm{l}=0.850 \\
\mathrm{w}=0.400\end{array}$ & $\begin{array}{c}\mathrm{l}=0.950 \\
\mathrm{w}=0.440\end{array}$ \\
\hline Vortex start time $(\mathrm{s})$ & 0.470 & 0.480 \\
\hline
\end{tabular}

are measured from the center of the electrode and from the bottom of the workpiece, respectively. Vortex size is the approximated size of the generated vortex in terms of length $(l)$ and width $(w)$. $l$ is measured along the $y$ direction, whereas $w$ is measured in the $x$ direction. Lastly, the vortex start time represents the moment when the vortex starts to appear in the lower side gap. It is measured from the beginning of the electrode jump.

According to the table, good, acceptable correspondence is obtained between the calculated and observed vortexes. Although the verification is performed only on the basis of the vortexes, similar acceptable results could be expected for the rest of the simulation using the same proposed CFD model and assumptions.

\section{Conclusion}

In this study, the dielectric fluid flow and the debris distribution in the machining gap during low and high electrode jumps were analyzed. The main conclusions obtained are as follows:

1. Electrode jump height affected the wall concavity of holes in deep EDM. The concavity is considered to be the total result of localized secondary discharges caused by debris-rich regions in the side gap.

2. The computational analysis of the dielectric fluid flow showed that the flow field pattern differs for low and high electrode jumps.
3. Simulation results of debris particle-fluid interaction showed that the amount of fluid pumped into and out of the gap by a low electrode jump is insufficient to eject the particles from the side gap properly. The redistributed debris particles inside the side gap formed debris-rich regions where secondary discharges are highly prone to occur just before electrical machining. Conversely, good disposal of debris particles was obtained with high electrode jumps.

4. A vortex was observed at the lower side gap during the end of the electrode jump in the simulation. This generated vortex captures some of the debris particles, by which the debris concentration in that specific region increases. Experiments were conducted to verify the simulation results for the vortex. It was confirmed that the results of the CFD model and calculations show good correlation with the experimental results.

A further statistical study on the debris distribution with various numbers and sizes of particles should improve the simulation. Similarly, adding bubble droplets into the present model and simulating the bubble-debrisfluid interaction will help greatly to understand the mechanism of debris movement for more precise and stable machining.

\section{References}

( 1 ) Akiyoshi, M. and Yusawa, T., Study of Sludge Behavior in Jump Movement of EDM, Annual Meeting of the Japan Society of Electrical Machining Engineers, (in Japanese), (2002), pp.97-98.

( 2 ) Kaneko, T. and Tsuchiya, M., Observation of Behavior of Machining Products in Die-Sinking EDM by Using High-Speed VTR, International Journal of Electrical Machining, No.2 (1997), pp.37-42.

( 3 ) Kunieda, M. and Yanatori, K., Study on Debris Movement in EDM Gap, International Journal of Electrical Machining, No.2 (1997), pp.43-49.

( 4 ) Lonardo, P.M. and Bruzzone, A.A., Effect of Flushing and Electrode Material on Die Sinking EDM, Annals of the CIRP, Vol.48, No.1 (1999), pp.123-126.

( 5 ) Masuzawa, T., Cui, X. and Taniguchi, N., Improved Jet Flushing for EDM, Annals of the CIRP, Vol.41, No.1 (1992), pp.239-242.

( 6 ) Masuzawa, T. and Heuvelman, C.J., A Self-Flushing Method with Spark-Erosion Machining, Annals of the CIRP, Vol.32, No.1 (1983), pp.109-111.

( 7 ) Kaneko, Y. and Yamada, H., Performance of Linear Motor Equipped Die-Sinking EDM, International Journal of Electrical Machining, No.5 (2000), pp.59-64.

( 8 ) Cetin, S., Uno, Y., Okada, A. and Tsuchiya, M., Effects of Electrode Jump Speed on EDM Characteristics, Annual Meeting of the Japan Society of Electrical Machining Engineers, (2001), pp.107-110.

(9) Cetin, S., Okada, A. and Uno, Y., Electrode Jump Motion in Linear Motor Equipped Die-Sinking EDM, Journal of Manufacturing Science and Engineering, Vol.125 (2003), pp.809-815. 
(10) Koenig, W., Weil, R., Wertheim, R. and Jutzler, W., The Flow Fields in the Working Gap with Electro Discharge Machining, Annals of the CIRP, Vol.25, No.1 (1977), pp.71-75.

(11) Kunieda, M. and Mori, M., Relation between Debris
Concentration and Discharge Gap Length in EDM Process, Proc. of Asian Electrical-Machining Symposium, (1995), pp.1-6.

(12) Computational Fluid Dynamics Software, Star-CD, Methodology Manual, (2001). 